\title{
BMJ Open A randomised controlled trial of aerobic exercise after transient ischaemic attack or minor stroke to prevent cognitive decline: the MoveIT study protocol
}

\author{
H M Boss, ${ }^{1}$ S M Van Schaik, ${ }^{1}$ I A Deijle, ${ }^{2}$ E C de Melker, ${ }^{3}$ B T J van den Berg, ${ }^{4}$ \\ E J A Scherder, ${ }^{5}$ W M J Bosboom, ${ }^{1} \mathrm{H}$ C Weinstein, ${ }^{1} \mathrm{R}$ M Van den Berg-Vos ${ }^{1}$
}

To cite: Boss HM, Van Schaik SM, Deijle IA, et al. A randomised controlled trial of aerobic exercise after transient ischaemic attack or minor stroke to prevent cognitive decline: the MovelT study protocol. BMJ Open 2014:4:e007065

doi:10.1136/bmjopen-2014007065

- Prepublication history for this paper is available online. To view these files please visit the journal online (http://dx.doi.org/10.1136/ bmjopen-2014-007065)

Received 1 November 2014 Accepted 3 December 2014

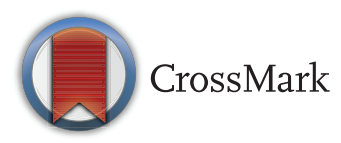

${ }^{1}$ Department of Neurology, Sint Lucas Andreas Hospital, Amsterdam, The Netherlands ${ }^{2}$ Department of Physical Therapy, Sint Lucas Andreas Hospital, Amsterdam,

The Netherlands

${ }^{3}$ Department of Cardiology, Sint Lucas Andreas Hospital Amsterdam, The Netherlands ${ }^{4}$ Department of Respiratory Medicine, Sint Lucas Andreas Hospital, Amsterdam,

The Netherlands

${ }^{5}$ Department of Clinical

Neuropsychology, VU

University, Amsterdam,

The Netherlands

Correspondence to RM Van den Berg-Vos; r.vandenberg-vos@slaz.nl

\section{ABSTRACT}

Introduction: Patients with transient ischaemic attack (TIA) or stroke are at risk for cognitive impairment and dementia. Currently, there is no known effective strategy to prevent this cognitive decline. Increasing evidence exists that physical exercise is beneficial for cognitive function. However, in patients with TIA or stroke who are at risk of cognitive impairment and dementia, only a few trials have been conducted. In this study, we aim to investigate whether a physical exercise programme (MovelT) can prevent cognitive decline in patients in the acute phase after a TIA or minor ischaemic stroke.

Methods and analysis: A single-blinded randomised controlled trial will be conducted to investigate the effect of an aerobic exercise programme on cognition compared with usual care. 120 adult patients with a TIA or minor ischaemic stroke less than 1 month ago will be randomly allocated to an exercise programme consisting of a 12-week aerobic exercise programme and regular follow-up visits to a specialised physiotherapist during the period of 1 year or to usual care. Outcome measures will be assessed at the baseline, and at the 1-year and 2-year follow-up. The primary outcome is cognitive functioning measured with the Montreal Cognitive Assessment (MoCA) test and with additional neuropsychological tests.

Secondary outcomes include maximal exercise capacity, self-reported physical activity and measures of secondary prevention.

Ethics and dissemination: The study received ethical approval from the VU University Amsterdam Ethics committee (2011/383). The results of this study will be published in peer-reviewed journals and presented at international conferences. We will also disseminate the main results to our participants in a letter.

Trial registration number: The Nederlands Trial Register NTR3884.

\section{INTRODUCTION}

Patients with transient ischaemic attack (TIA) or ischaemic stroke have an increased risk of developing cognitive impairment and

\section{Strengths and limitations of this study}

- This randomised controlled trial will provide new evidence on the effects of physical exercise on cognition in patients in the acute phase after transient ischaemic attack or minor stroke.

- Assessments will be performed by personnel blinded to the treatment allocation.

- One study site may affect the generalisability.

- The number of patients in our sample is not sufficient to investigate hard outcomes (mortality, incidence of cardiovascular events).

dementia. ${ }^{1}{ }^{2}$ Cognitive impairment not only occurs in patients with major stroke, but also in patients after TIA or minor stroke. ${ }^{3-5}$ In a meta-analysis of 7511 patients, $10 \%$ developed new dementia after the first stroke and more than a third had dementia after a recurrent stroke. ${ }^{1}$ In patients with stroke, the prevalence of mild cognitive impairment varies between $20 \%$ and $90 \% .^{26}$ In addition, multiple studies demonstrated that the occurrence of cognitive impairment or dementia after stroke predicts death and dependency. ${ }^{7-10}$ Currently, there is no known effective strategy to prevent cognitive decline or dementia in these patients.

Physical activity has been associated with a decreased risk for dementia and cognitive decline. ${ }^{11}{ }^{12}$ In healthy elderly individuals, there is evidence that a physical exercise programme is associated with an improvement in cognitive performance. ${ }^{13}$ Current stroke guidelines advise at least $30 \mathrm{~min}$ of moderateintensity physical exercise 3-7 times a week for patients with TIA or ischaemic stroke who are capable of engaging in physical activity. ${ }^{14}$ For patients after myocardial infarction, a physical exercise programme is standard care and a part of cardiac rehabilitation, and this exercise programme is associated with a reduced mortality. ${ }^{15}$ 
In patients with vascular diseases, conflicting results have been found with regard to the association between physical activity and cognition. One cohort study found that physical activity was associated with better preservation of cognitive function; ${ }^{16}$ another recent study could not confirm this association. ${ }^{17}$ Until now, three observational studies and only one randomised controlled trial have assessed the effect of a physical exercise programme on cognition in patients with a TIA or stroke. ${ }^{18-21}$ This randomised controlled trial only included patients who had a stroke more than 6 months ago and found an improvement in motor learning. ${ }^{19}$ No randomised controlled trials have been performed in the acute phase following a TIA or stroke.

Previously, we demonstrated the safety and feasibility of an exercise programme in the acute phase after a TIA or a minor ischaemic stroke. ${ }^{22}$ The aim of this larger study is twofold: (1) to investigate whether a physical exercise programme (MoveIT) can prevent cognitive decline in patients after a TIA or minor ischaemic stroke, and (2) to investigate the effect of this programme on cardiorespiratory fitness and the attainment of secondary prevention targets.

\section{METHODS AND ANALYSIS}

\section{Study design and setting}

MoveIT is a two parallel group, single-centre, singleblinded, randomised controlled trial to investigate the effects of an aerobic exercise programme on cognition in patients in the acute phase after TIA or minor ischaemic stroke (figure 1). The study will be conducted in the Sint Lucas Andreas Hospital, a district hospital in Amsterdam with a specialised stroke unit. Participants will be randomly assigned to a control or intervention group. The control group will receive the usual care for patients after TIA or minor ischaemic stroke, consisting of two or three visits to the outpatient clinic. The intervention group will participate in the MoveIT programme, which consists of a 12-week aerobic exercise programme and follow-up visits to a specialised physiotherapist every 3 months during the period of 1 year. Study procedures were approved by local university and hospital research ethics committees. Informed written consent will be obtained from all participants. The study has been registered at the trial registration (The Nederlands Trial Register-NTR3884).

\section{Objectives}

Primary objective. To investigate the effect of a physical exercise programme (MoveIT) on cognition in patients in the acute phase after a TIA or minor ischaemic stroke, compared with participation in usual care.

Secondary objectives:

- To investigate the effect of a physical exercise programme on cardiorespiratory fitness, measured by maximal exercise capacity.
- To investigate the effect of a physical exercise programme on the attainment of secondary prevention targets, defined as the combination of prescribed antithrombotic therapy (antiplatelet agents or oral anticoagulants) and achievement of blood pressure $(<140 / 90 \mathrm{~mm} \mathrm{Hg})$ and low-density lipoprotein cholesterol (LDL-c; $<100 \mathrm{mg} / \mathrm{dL}$ ) targets, as recommended in international guidelines. ${ }^{23}$

\section{Participants}

\section{Inclusion criteria}

Patients are eligible if they (1) are at least 18 years old, (2) present with a TIA or minor ischaemic stroke as defined by the National Institutes of Health Stroke Scale (NIHSS) score $\leq 3,{ }^{24}$ (3) had the onset of signs and symptoms less than 1 month ago, (4) are able to walk independently and (5) are discharged from hospital without need for further rehabilitation.

\section{Exclusion criteria}

Patients will be excluded if they have (1) dementia or a Mini-Mental State Examination (MMSE) score <24, (2) aphasia or the inability to speak Dutch, (3) cardiopulmonary contraindication for physical exercise and exercise testing outlined by the American College of Sports Medicine (ACSM $)^{25}$ or (4) chronic disease with an expected survival of less than 2 years.

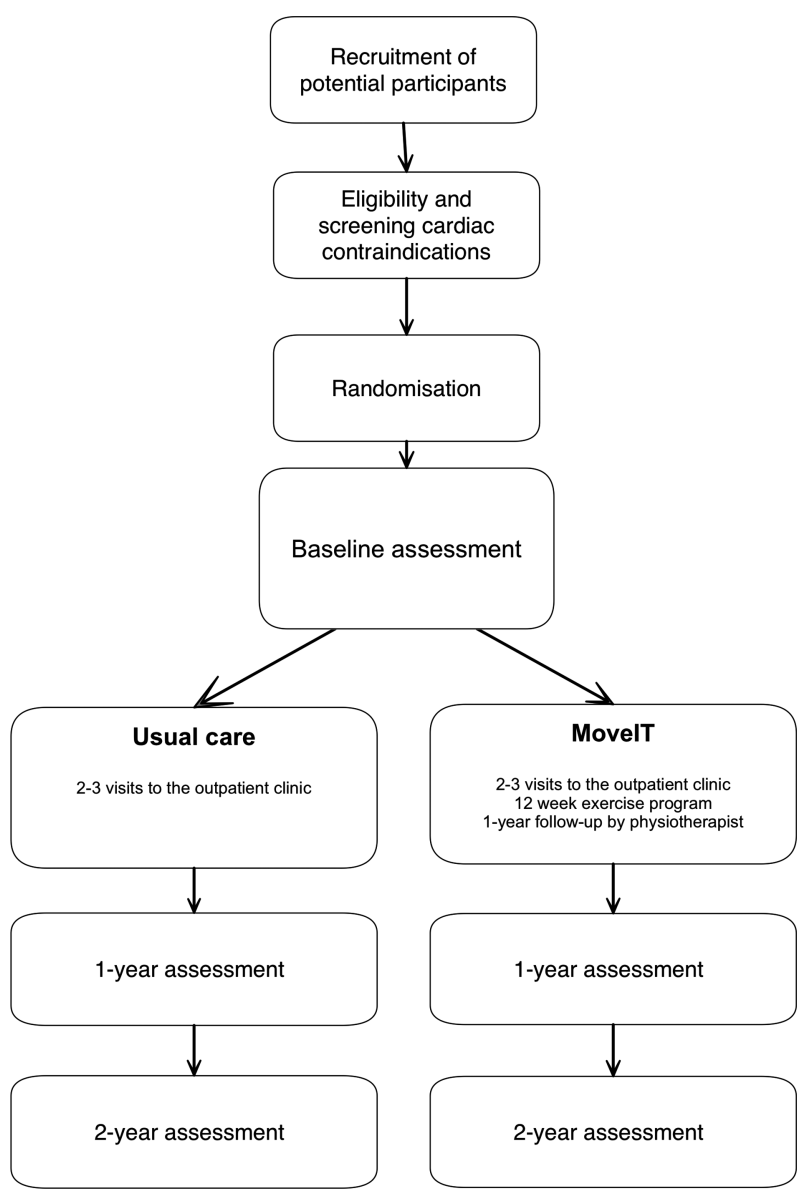

Figure 1 Flow chart of trial design. 


\section{Recruitment}

Consecutive patients with a TIA or a minor ischaemic stroke admitted to the stroke unit or evaluated in the outpatient clinic or emergency room of the Sint Lucas Andreas Hospital will be given information about the trial. Patients who are interested to participate will be approached with further information and screened for cardiac contraindication using a cardiac checklist. This cardiac checklist has been developed by our study group and consists of items concerning the patient's medical history, disease history, physical and ancillary (ECG) investigations. Before randomisation, all potential participants with a positive cardiac checklist will be examined by a cardiologist to exclude possible cardiac contraindication for maximal exercise testing or a physical exercise programme. After exclusion of cardiac contraindications or a negative cardiac checklist, written informed consent will be obtained. If there are indications of pulmonary disease, patients will be referred to a pulmonologist to optimise the pulmonary function prior to the start of the programme.

\section{Randomisation}

Consenting participants will be allocated for treatment using block randomisation with a block size of two. The allocation sequence will be generated by coin tossing. Randomisation will be conducted independently using sealed opaque envelopes.

\section{Intervention}

Participants randomly assigned to the intervention group will participate in the MoveIT programme. This programme consists of a 12-week aerobic exercise programme, combined with regular follow-up visits to a specialised physiotherapist during the period of 1 year. During the aerobic exercise programme, participants will receive two $1 \mathrm{~h}$ exercise sessions per week supervised by two specialised physiotherapists. These sessions consist of aerobic exercise and strength training. The exercise intensity will be based on the individual's maximal heart rate and the maximal power achieved during the maximal exercise test. During the programme, the exercise intensity will gradually be increased. Participants will start with home-based aerobic exercise and will gradually increase this frequency to three times a week. Participants will keep a $\log$ of these activities and will discuss their progress with the physiotherapists during the exercise sessions and during the follow-up visits after the completion of the exercise programme.

After the completion of the physical exercise programme, follow-up consists of a total of three visits to the physiotherapist during the period of 1 year. During these appointments, participants will receive motivational interviewing-based counselling by physiotherapists in order to motivate them to maintain an active lifestyle. Participating physiotherapists received a motivational interviewing training and have considerable experience with this technique.

\section{Control group}

Participants in both groups will continue to receive usual stroke care. Usual care consists of a total of 2-3 follow-up visits to the outpatient clinic for 3 months after the TIA or stroke. During these appointments, the motivational interviewing-based counselling will be focused on the attainment of secondary prevention and the improvement of lifestyle factors and physical activity.

\section{Data collection and outcomes measures}

Outcome measures will be assessed at the baseline, and at the 1-year and 2-year follow-up by personnel blinded to the treatment allocation. Data will be recorded on standardised forms and entered into a secured Access database with Structured Query Language (SQL) that contains quality control checks (eg, range checks, notification of missing data).

\section{Cognitive functioning}

The primary end point is global cognitive functioning measured using the Montreal Cognitive Assessment (MoCA) test, ${ }^{26}$ which has been validated to measure cognitive decline in patients after stroke. ${ }^{27}$

In addition, a standardised neuropsychological examination will be performed which consists of attention, verbal and visual memory, and executive functioning tests. Verbal memory will be assessed using the Dutch version of the California Verbal Learning Test (VGLT) ${ }^{28}$ Non-verbal memory will be assessed with the recall of the Rey-Osterrieth Complex Figure Test. ${ }^{29}$ Executive functioning will be assessed using the Key Search and Rule Shift Cards from the Behavioural Assessment of the Dysexecutive Syndrome (BADS),${ }^{30}$ letter fluency and categorical fluency, ${ }^{31}$ difference in time between part A and B of Trail Making Test, ${ }^{32}$ the interference score of the Stroop Color Word Test ${ }^{33}$ and the Stop Signal Task of the CANTAB. ${ }^{34}$ Attention will be assessed with the digit span forwards and backwards of the Wechsler Adult Intelligence Scale (WAIS). ${ }^{35}$ In addition, participants will fill out a validated questionnaire measuring cognitive symptoms (Cognitive Failures Questionnaire). ${ }^{36}$

Baseline composite z-scores for the cognitive domains: attention, memory and executive functioning will be calculated by averaging the z-scores (raw individual test score at baseline minus the mean test score divided by the SD). Follow-up composite z-scores will be calculated for the cognitive domains: attention, memory and executive functioning by averaging the z-scores (raw individual test score at follow-up minus mean baseline test score divided by the SD of the baseline test score). These composite z-scores will be used in the further analysis.

\section{Measures of physical function}

Maximal exercise capacity $\left(\mathrm{VO}_{2 \max }\right)$ or maximal oxygen consumption $(\mathrm{mL} / \mathrm{kg} / \mathrm{min})$ is used as the measure of cardiorespiratory fitness. A symptom-limited ramp exercise test will be performed on a Jaeger cycle ergometer. During these tests, continuous ECG monitoring will be 
performed and the blood pressure will be measured every minute. Oxygen consumption $\left(\mathrm{VO}_{2}\right)$ will be continuously measured using a metabolic measurement system, which is performed by a breath-by-breath gas analysis (Oxycon Pro, Jaeger). The testing protocol will be adjusted to the capabilities of the patient. ${ }^{25}$ Exercise will be terminated if participants are fatigued or earlier if they fulfil the ACSM's guidelines for 'Indications for terminating Exercise Testing'. ${ }^{25}$ A cardiologist and pulmonologist will review all results of the exercise tests. In case of ECG-abnormalities, a cardiologist will examine the participant to exclude cardiac disease. The maximal value obtained will be considered as the $\mathrm{VO}_{2 \max }$.

The amount of self-reported physical activity will be measured using the Physical Activity Scale for the Elderly (PASE) questionnaire. ${ }^{37} 38$ This questionnaire has also been validated for the elderly patients in the Dutch population. ${ }^{38}$

\section{Measures of secondary prevention}

Secondary prevention will be measured as the number of participants who achieve the composite end point of optimal medical therapy, defined as the combination of prescribed antithrombotic therapy (antiplatelet agents or oral anticoagulants) and achievement of both blood pressure $(<140 / 90 \mathrm{~mm} \mathrm{Hg})$ and LDL-c $(<100 \mathrm{mg} / \mathrm{dL})$ targets. These cut-off points are based on current guidelines. ${ }^{23}$ Other end points are the individual components of the composite end point of optimal medical therapy, number of participants not smoking (self-reported), alcohol consumption (self-reported), body mass index and waist circumference.

\section{Recurrent vascular events}

All recurrent vascular events during the follow-up period of 2 years will be recorded. The trial is not sufficiently powered to determine the effect of the MoveIT programme in reducing recurrent TIA, stroke or cardiac events. Thus, it will be exploratory in this regard.

\section{Other}

Mental health and fatigue will be assessed using the Hospital Anxiety and Depression Scale (HADS) ${ }^{39}$ and the Fatigue Severity Scale. ${ }^{40}$

\section{Covariates}

Imaging

At baseline, in all participants a non-contrast head CT scan will be performed as part of their routine stroke workup. In addition, in all participants without contraindications, a brain MRI will be performed after informed consent. The MR investigations will be performed on a 1.5-Tesla scanner. The protocol consists of sagittal T1-weighted (repetition time (TR)/echo time (TE): $1940 / 3.08 \mathrm{~ms}$ ), sagittal fluid-attenuated inversion recovery (TR/TE 6000/358 ms), transversal T2-weighted (TR/ TE 5000/96 ms), transversal T2-weighted gradient echo (TR/TE 800/26 ms) and diffusion-weighted imaging
(TR/TE 4100/102 ms). Imaging will be used to evaluate markers of small vessel disease, brain atrophy and carotid calcifications.

\section{Apolipoprotein E}

APOE genotype, a genetic risk factor for Alzheimer disease, will be performed after DNA isolation from 10 ml EDTA blood by using the LightCycler APOE mutation detection method (Roche Diagnostics $\mathrm{GmbH}$ ) and coded as $A P O E \varepsilon 4$ or no $\varepsilon 4$.

\section{Other}

Pre-existent cognitive impairment will be assessed using the Informant Questionnaire for Cognitive Decline in Elderly (IQCODE) ${ }^{41}$ Premorbid intellectual functioning will be assessed using the Dutch version of the National Adult Reading Test (DART) ${ }^{42}$

\section{Power calculation and sample size}

Global cognition, assessed with the MoCA test, was selected as the outcome variable to calculate sample size. ${ }^{2627}$ Using estimates obtained from the literature ${ }^{4344}$ and our previously performed pilot study, ${ }^{22}$ a sample size of 52 patients in each group is needed to reach a power of $80 \%$ to detect a difference in means in the MoCA score of 1.5 point, assuming a SD of 2.7 using a two-sample $\mathrm{t}$ test with a 0.05 two-sided significance level. We increased the sample size to 60 patients in each group to anticipate for potential dropouts.

\section{Analysis}

Baseline demographic and clinical characteristics of participants in the intervention and control groups will be compared using two-sample $\mathrm{t}$ tests (continuous data) and $\chi^{2}$ analysis (nominal data). Non-parametric methods will be used when assumptions of normality are violated.

Primary analyses will be unadjusted, following the intention-to-treat principle. For the primary end point, the change in MoCA between the baseline and the 2-year follow-up will be calculated. Depending on the normality distribution, the change scores will be compared using either Wilcoxon rank-sum tests or twosample $t$ tests with significance level of 0.05 .

In the secondary analyses, we will assess the effect on our secondary outcome measures. We will calculate the change between the baseline and the 2 years continuous data, and this change score will be analysed using either Wilcoxon rank-sum tests or two-sample $\mathrm{t}$ tests. We will perform logistic regression for the outcome measures with a dichotomous outcome, such as attainment of measures for secondary prevention using the data at the 2-year follow-up as the dependent variable and correcting for baseline using the baseline data as covariate.

A repeated-measures mixed design will be used to estimate the treatment effects (2 groups at 3 time points), using the variables measured at the baseline, and at the 1-year and 2-year follow-up that conform to the 
assumptions of normality. Level of significance will be $<0.05$. Data will be analysed using SPSS (V.20.0).

\section{Ethics and dissemination}

All minor and major amendments to the protocol will be approved by the Ethics committee. A signed and dated informed consent form will be required from all participants. The risks and benefits of participation will be explained and all potential participants are free to decline to participate in the trial. All patient-level data accessed in this study will be stripped of personal identifiers. To guarantee the confidentiality of the participants, only the authors will have access to the data during the study.

We will disseminate the results of our study via presentations at international conferences and publications in peer-reviewed journals.

Contributors HMB, SMVS and RMVdB-V contributed to the conception and design of the study protocol and the drafting of this work, have given their approval for publication and are accountable for all aspects of this work. IAD, ECdM, BTJvdB, EJAS, WMJB, and HCW contributed to the design of the study protocol, revised this work critically for important intellectual content, have given their approval for publication and are accountable for all aspects of this work.

Funding This work was supported by ZonMW (project number 60-82600-98-8338), Roomsch Catholijk Oude Armen Kantoor (project number 2010.101) and the 'Innovatiefonds' of the Sint Lucas Andreas Hospital 2010 (Motives MovelT 2010).

Competing interests None.

Ethics approval The study received ethical approval from the VU University Amsterdam Ethics Committee (2011/383).

Provenance and peer review Not commissioned; externally peer reviewed.

Open Access This is an Open Access article distributed in accordance with the Creative Commons Attribution Non Commercial (CC BY-NC 4.0) license, which permits others to distribute, remix, adapt, build upon this work noncommercially, and license their derivative works on different terms, provided the original work is properly cited and the use is non-commercial. See: http:// creativecommons.org/licenses/by-nc/4.0/

\section{REFERENCES}

1. Pendlebury ST, Rothwell PM. Prevalence, incidence, and factors associated with pre-stroke and post-stroke dementia: a systematic review and meta-analysis. Lancet Neurol 2009;8:1006-18.

2. Gottesman RF, Hillis AE. Predictors and assessment of cognitive dysfunction resulting from ischaemic stroke. Lancet Neurol 2010;9:895-905.

3. Moran GM, Fletcher B, Feltham MG, et al. Fatigue, psychological and cognitive impairment following transient ischaemic attack and minor stroke: a systematic review. Eur J Neurol 2014;21:1258-67.

4. van Rooij FG, Schaapsmeerders P, Maaijwee NAM, et al. Persistent cognitive impairment after transient ischemic attack. Stroke 2014;45:2270-4.

5. Dong Y, Slavin MJ, Chan BP-L, et al. Improving screening for vascular cognitive impairment at three to six months after mild ischemic stroke and transient ischemic attack. Int Psychogeriatr 2014:26:787-93.

6. Douiri A, Rudd AG, Wolfe CDA. Prevalence of poststroke cognitive impairment: South London Stroke Register 1995-2010. Stroke 2013;44:138-45.

7. Narasimhalu K, Ang S, De Silva DA, et al. The prognostic effects of poststroke cognitive impairment no dementia and domain-specific cognitive impairments in nondisabled ischemic stroke patients. Stroke 2011;42:883-8.

8. Tatemichi TK, Paik M, Bagiella E, et al. Dementia after stroke is a predictor of long-term survival. Stroke 1994;25:1915-19.
9. Brodaty $\mathrm{H}$, Altendorf $\mathrm{A}$, Withall $\mathrm{A}$, et al. Mortality and institutionalization in early survivors of stroke: the effects of cognition, vascular mild cognitive impairment, and vascular dementia. J Stroke Cerebrovasc Dis 2010;19:485-93.

10. Hobson P, Meara J. Cognitive function and mortality in a community-based elderly cohort of first-ever stroke survivors and control subjects. J Stroke Cerebrovasc Dis 2010;19:382-7.

11. Buchman AS, Boyle PA, Yu L, et al. Total daily physical activity and the risk of $A D$ and cognitive decline in older adults. Neurology 2012;78:1323-9.

12. Weuve J, Kang JH, Manson JE, et al. Physical activity, including walking, and cognitive function in older women. JAMA 2004;292:1454-61.

13. Angevaren M, Aufdemkampe G, Verhaar HJJ, et al. Physical activity and enhanced fitness to improve cognitive function in older people without known cognitive impairment. Cochrane Database Syst Rev 2008;3:CD005381.

14. Gordon NF. Physical activity and exercise recommendations for stroke survivors: an American Heart Association Scientific Statement from the Council on Clinical Cardiology, Subcommittee on Exercise, Cardiac Rehabilitation, and Prevention; the Council on Cardiovascular Nursing; the Council on Nutrition, Physical Activity, and Metabolism; and the Stroke Council. Stroke 2004;35:1230-40.

15. Taylor RS, Brown A, Ebrahim S, et al. Exercise-based rehabilitation for patients with coronary heart disease: systematic review and meta-analysis of randomized controlled trials. Am J Med 2004;116:682-92.

16. Vercambre MN, Grodstein F, Manson JE, et al. Physical activity and cognition in women with vascular conditions. Arch Intern Med 2011;171:1244-50.

17. Kooistra M, Boss HM, van der Graaf $Y$, et al. Physical activity, structural brain changes and cognitive decline. The SMART-MR study. Atherosclerosis 2014;234:47-53.

18. Marzolini S, Oh P, Mcllroy W, et al. The effects of an aerobic and resistance exercise training program on cognition following stroke. Neurorehabil Neural Repair 2013;27:392-402.

19. Quaney BM, Boyd LA, McDowd JM, et al. Aerobic exercise improves cognition and motor function poststroke. Neurorehabil Neural Repair 2009;23:879-85.

20. Kluding PM, Tseng BY, Billinger SA. Exercise and executive function in individuals with chronic stroke. J Neurol Phys Ther 2011;35:11-17.

21. Rand D, Eng JJ, Liu-Ambrose T, et al. Feasibility of a 6-month exercise and recreation program to improve executive functioning and memory in individuals with chronic stroke. Neurorehabil Neural Repair 2010;24:722-9.

22. Boss HM, Van Schaik SM, Deijle IA, et al. Safety and feasibility of post-stroke care and exercise after minor ischemic stroke or transient ischemic attack: MotiveS \& MovelT. NeuroRehabilitation 2014;34:401-7.

23. Furie KL, Kasner SE, Adams RJ, et al. Guidelines for the prevention of stroke in patients with stroke or transient ischemic attack: a guideline for healthcare professionals from the American Heart Association/American Stroke Association. Stroke 2010;42:227-76.

24. Brott T, Adams HP, Olinger CP, et al. Measurements of acute cerebral infarction: a clinical examination scale. Stroke 1989;20:864-70.

25. American College of Sports Medicine. ACSM's guidelines for exercise testing and prescription, ed 8. Baltimore: Wolters Kluwer, 2010.

26. Nasreddine ZS, Phillips NA, Bédirian V et al. The Montreal Cognitive Assessment, MoCA: a brief screening tool for mild cognitive impairment. J Am Geriatr Soc 2005;53:695-9.

27. Pendlebury ST, Cuthbertson FC, Welch SJV, et al. Underestimation of cognitive impairment by Mini-Mental State Examination versus the Montreal Cognitive Assessment in patients with transient ischemic attack and stroke: a population-based study. Stroke 2010;41:1290-3.

28. Delis DC, Kramer JH, Kaplan E, et al. California verbal learning test: adult version, manual. San Antonio, Tex: Psychological Corp, 1987.

29. Rey A. L'examen psychologique dans les cas d'encéphalopathie traumatique; Les problèmes. Arch Psychol 1941;28:215-85.

30. Wilson BA, Alderman N, Burgess PW, et al. Behavioural assessment of the dysexecutive syndrome. Bury St. Edmunds, UK Thames Valley Test Company, 1996.

31. Lezak MD. Neurological assessment. 4th ed. New York: Oxford University Press, 2004.

32. Reitan RM. Validity of the Trail Making Test as an indicator of organic brain damage. Percept Mot Skills 1958;8:271-6.

33. Stroop JR. Studies of interference in serial verbal reactions. J Exp Psychol 1935;18:643-62. 
34. Sahakian BJ, Owen AM. Computerized assessment in neuropsychiatry using CANTAB: discussion paper. J R Soc Med 1992;85:399-402.

35. Wechsler D. Wechsler Adult Intelligence Scale. New York: Psychological Corp, 1955.

36. Broadbent DE, Cooper PF, FitzGerald P, et al. The Cognitive Failures Questionnaire (CFQ) and its correlates. Br J Clin Psychol 2009;21:1-16.

37. Washburn RA, Smith KW, Jette AM, et al. The Physical Activity Scale for the Elderly (PASE): development and evaluation. J Clin Epidemiol 1993:46:153-62.

38. Schuit AJ, Schouten EG, Westerterp KR, et al. Validity of the Physical Activity Scale for the Elderly (PASE): according to energy expenditure assessed by the doubly labeled water method. J Clin Epidemiol 1997;50:541-6.

39. Zigmond AS, Snaith RP. The hospital anxiety and depression scale. Acta Psychiatr Scand 1983;67:361-70.
40. Krupp LB, LaRocca NG, Muir-Nash J, et al. The fatigue severity scale. Application to patients with multiple sclerosis and systemic lupus erythematosus. Arch Neurol 1989;46:1121-3.

41. Jorm AF, Jacomb PA. The Informant Questionnaire on Cognitive Decline in the Elderly (IQCODE): socio-demographic correlates, reliability, validity and some norms. Psychol Med 1989;19:1015-22.

42. Schmand B, Geerlings MI, Jonker C, et al. Reading ability as an estimator of premorbid intelligence: does it remain stable in emergent dementia? J Clin Exp Neuropsychol 1998;20:42-51.

43. Sachdev PS, Brodaty H, Valenzuela MJ, et al. Progression of cognitive impairment in stroke patients. Neurology 2004:63:1618-23.

44. Popović IM, Šerić V, Demarin V. Mild cognitive impairment in symptomatic and asymptomatic cerebrovascular disease. J Neurol Sci 2007;257:185-93. 